\title{
THE BLACKENING OF A PHOTOGRAPHIC PLATE AS A FUNCTION OF INTENSITY OF LIGHT AND TIME OF EXPOSURE.
}

By P. S. Helmick.

Synopsis.

Blackening of Photographic Plates as a Function of Light Intensity, Wave-length and Exposure.-Three emulsions, a slow one (Seed 23), a rapid one (Seed $27 X$ ) and a very rapid one (Seed Graflex) were coated on plate glass and illuminated for various lengths of time with various intensities of monochromatic light of wave-length $45^{\circ}, 55^{\circ}$ and $650 \mu \mu$. The results are given in curves and are found to be well repiesented, for a given emulsion and wave-length, by the empirical equation for blackening, $D=(\mathrm{I} / a) \log \left[b-(b-\mathrm{I}) e^{-c t}\right]$, where $\log c=A+B \log I+c \log ^{2} I$, and $a, b, A, B$ and $C$ are constants. A graphical method of determining these constants employing a monographic chart is described. Schwarzschild's exponent $p$ is shown to be equal to $(B+C \log I)^{-1}$, and is found to vary from 0.7 to 1.95 according to the emulsion, wave-length and intensity. In using the photographic method of measuring the intensity of a source of light, it is obviously important to determine the constants of the equation for the blackening of the emulsion for the proper wavelength. The method of doing this is explained in detail.

$\mathrm{T}$

HE object of this research has been the expression of the blackening of a photographic plate as a function of the intensity of monochromatic light and time of exposure to light, other factors being constant.

Such an expression finds practical application in photometry of monochromatic sources. By exposing a plate to a light source of unknown intensity for any known length of time, measurement of the resulting blackening will enable the unknown intensity of light to be calculated.

\section{Apparatus and Materials.}

The plates used in the work were coated on special plate glass, and the variations of density due to unevenness of coating were of the order of I per cent. Three emulsions were used: " $A$ " a slow emulsion (Seed 23), " $B$ " a rapid emulsion (Seed $27 X$ ), and " $C$ " a very rapid emulsion (Seed Graflex).

The source of light was a Hilger Monochromatic Illuminator. Light from a constant-voltage tungsten lamp was focussed upon the collimating slit of the illuminator, and was again focused upon the second slit of the instrument, before emerging into the exposing box.

Variations in intensity of light were obtained by varying the distance 
between the plate and the second slit of the illuminator, assuming the inverse square law, and applying the necessary correction for finite width of source. ${ }^{1}$

Intensities were measured in watts/sq. meter by a thermopile calibrated against a standard lamp whose radiation was certified to by the Bureau of Standards.

Exposures were made by cutting one of the $5 \times 7$ plates into 30 strips, and exposing 12 of these, the remaining strips being used to obtain the amount of fog, as explained later. Three wave-lengths of light were used: $450 \mu \mu, 550 \mu \mu$, and $650 \mu \mu$. A hand-operated shutter was mounted in front of the slit of the illuminator, and by listening to a telephone receiver clicking seconds, exposures consisting of any integral number of seconds could easily be made.

Plates were developed at a practically constant temperature in a developer compounded after Brush's formula. ${ }^{2}$ The maximum variation in temperature during development of the plates which make up one of the sets of curves shown later on was never more than $I^{\circ} .8 \mathrm{C}$., generally it was only a few tenths of a degree. A time of development of 6 minutes at $23^{\circ} \mathrm{C}$. was selected as standard. This variation in temperature was corrected for by the use of Watkins' formula ${ }^{3}$

$$
r=\left(s_{1} / s_{2}\right)^{\frac{10}{t_{2}-t_{1}}}
$$

where $r=$ temperature coefficient, $t_{1}=$ low temperature, $t_{2}=$ high temperature, $s_{1}=$ time of development at $t_{1}$, and $s_{2}=$ time of development at $t_{2}{ }^{4}$

The density of a photographic plate is defined as $\log _{10} 0$, where 0 , the opacity, is the ratio of the incident to the transmitted light. Densities were measured in a modification of Lemon's spectro-photometer, ${ }^{5}$ in which the prism is replaced by two mirrors inclined to one another so as to reflect two beams of light into the observing telescope. The density corresponding to an angle $\theta$ between transmitting planes of the nicols when rotated so as to cut down one light beam to the same intensity which the interposed plate of unknown density cuts down the other light beam is given by the expression $\log _{10} \operatorname{Sec}^{2} \theta$.

1 See Bull. Bur. Standards, 3, 8I; I907.

2 Phys. Rev., 31, 243; 1910.

3 The A. Photographer and Photo. News, London, May I7, r9ro.

4 According to Watkins, the value of " $r$ " for any hydroquinone developer is 2.25 , so this value was accordingly taken for the " $r$ " of the hydroquinone developer used in this work. For example, if the temperature were $22^{\circ} \mathrm{C}$., the time of development allowed was given by the solution of the equation

5 Astrophys. Journ., 39, 204; I914.

$$
2.25=\left[\frac{S_{1}}{6}\right] \frac{10}{33-22} .
$$


Densities were measured with light of $535 \mu \mu$, obtained by placing two "monochromatic" color filters in front of the observing telescope. Density readings on a single plate were repeated until the probable error of the mean was reduced to a very small value.

\section{Brand $A$.}

Wave-length $550 \mu \mu$. Distance $226.2 \mathrm{~cm} . \quad 1.53 \times 10^{-4}$ watts $/ \mathrm{sq} . \mathrm{m}$. Temperature $22^{\circ}$.o. Time Devel. $6^{\mathrm{m}} 30^{\mathrm{s}}$.

\begin{tabular}{|c|c|c|c|c|c|}
\hline Plate. & Time. & $D$ & Plate. & Time. & $D$ \\
\hline 1500 . & & 0.35 & $1514 \ldots$ & & 0.29 \\
\hline $1501 \ldots$ & $15^{\mathrm{m}}$ & 6.31 & $1515 \ldots$ & $15^{s}$ & 0.32 \\
\hline 1502 . & & 0.39 & $1516 \ldots$ & & 0.26 \\
\hline $1503 \ldots \ldots$ & $8^{m}$ & 4.03 & $1517 \ldots \ldots$ & $8^{s}$ & 0.25 \\
\hline $1504 \ldots \ldots \ldots$ & & 0.37 & $1520 \ldots$ & & 0.25 \\
\hline $1505 \ldots$ & $4^{\mathrm{m}}$ & 2.88 & $1521 \ldots$ & $4^{s}$ & 0.27 \\
\hline $1506 \ldots$ & & 0.42 & $1522 \ldots$ & & 0.27 \\
\hline $1507 \ldots$ & $2^{\mathrm{m}}$ & 1.91 & $1523 \ldots$ & $2^{s}$ & 0.28 \\
\hline $1510 \ldots$ & & 0.34 & $1524 \ldots$ & & 0.27 \\
\hline $1511 \ldots \ldots \ldots$ & $1^{\mathrm{m}}$ & 1.35 & $1525 \ldots \ldots \ldots$ & $1^{\text {s }}$ & 0.26 \\
\hline $1512 \ldots \ldots \ldots$ & & 0.31 & $1526 \ldots \ldots \ldots$ & & 0.24 \\
\hline $1513 \ldots \ldots \ldots$ & $30^{s}$ & 0.58 & $1527 \ldots \ldots \ldots$ & & 0.28 \\
\hline
\end{tabular}

The preceding table is a sample of one set of exposures. All of the separate small plates were cut from the same $5 \times 7$ plate. The small plates were numbered to show how they were cut with respect to the original plate. For example I5I3 lay adjacent to I5I2, I5I4, I5O3 and $15^{23}$ in the original plate. The even numbers were not exposed, and were used to obtain the fog. These unexposed fog strips were developed along with the exposed plates in order to find the blackening of the exposed plates which was not due to exposure to light. This fog density was then subtracted from the densities of the exposed plates in the calculations and curves.

The plates were of brand " $A$," exposed to light of wave-length $550 \mu \mu$ at a distance of $226.2 \mathrm{~cm}$. from the slit of the monochromatic illuminator, the flow of energy on the plate being $1.53 \times 10^{-4}$ watts $/ \mathrm{sq} . \mathrm{m}$. The plates were developed at a temperature of $22^{\circ} .0$ for a time of $6^{\mathrm{m}} 30^{\mathrm{s}}$.

The table gives the plate number, the time of exposure, and the mean of the density determinations of the plate.

\section{THEORETICAL.}

In 1890, Hurter and Driffield ${ }^{1}$ developed an equation of the following form for the density $D$ of a photographic plate exposed for a time $t$ to light of constant intensity $I$ :

$$
D=\alpha \log _{e}\left[\beta-(\beta-1) e^{-\gamma I t}\right],
$$

${ }^{1}$ Journ. Soc. Chem. Ind., 9, 455; 1890. 
where $\alpha, \beta$, and $\gamma$ are parameters dependent upon the character of the plate and the developer, $-\beta$ is equal to the opacity of the unexposed plate. They assumed that the amount of silver made developable by the light was proportional to $I \cdot t$, and that only the light absorbed by the unaltered silver particles contributed to the building up of the latent image.

This expression would lead to the so-called "Reciprocity Law" of Bunsen and $\operatorname{Roscoe}^{1}(I \cdot t$ equals constant for equal blackening) which Abney, ${ }^{2}$ Miethe, ${ }^{3}$ Eder, ${ }^{4}$ Michalke and Schiener, Schwarzschild, ${ }^{5}$ Kron, ${ }^{6}$ Lemon, ${ }^{7}$ and others have shown to be only a very rough approximation to the facts.

However, for a constant intensity of light, the expression gives a very good relationship between the density of the plate and the time of exposure, as both Hurter and Driffield, and Sheppard and Mees, ${ }^{8}$ have shown.

In this work the equation of Hurter and Driffield is made use of in the following way:

Given a set of curves showing the relationship between density and time of exposure for intensities of light $I_{1}=k_{1}, I_{2}=k_{2}, \cdots, I_{n}=k_{n}$.

We will rewrite the equation of Hurter and Driffield in the form:

$$
D=\mathrm{I} / a \log _{e}\left[b-(b-\mathrm{I}) e^{-c t}\right] .
$$

Then for the curves $I_{1}, I_{2}, \cdots, I_{n}$, we can find values of the parameters $a, b, c_{1}, c_{2}, \cdots, c_{n}$, respectively which will give us this set of $n$ curves. But $c$ is a function of $I$, and if we can find this function, we can write our equation in the form:

$$
D=\mathrm{I} / a \log _{e}\left[b-(b-\mathrm{I}) e^{-f(I) \times t}\right]
$$

and thus the density is completely determined in terms of the intensity of light and time of exposure.

Hurter and Driffield gave no hint as to the method of determination of the parameters $a, b$, and $c$, and Sheppard and Mees give a method applicable only when $a=\mathrm{I}$. Two methods are here developed which may be used to find the values of these parameters from a given experimental curve.

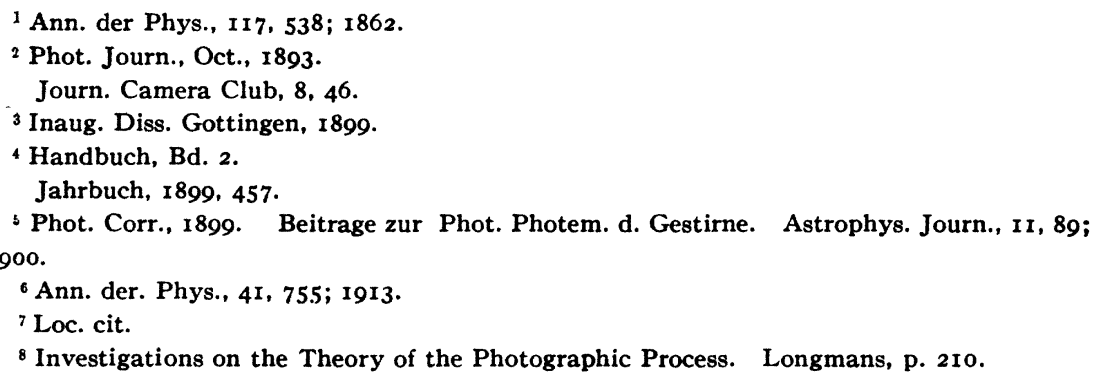


Assuming that the experimental curve is of the form given above, select four points, $\left(t_{1}, D_{1}\right),\left(t_{2}, D_{2}\right),\left(t_{3}, D_{3}\right)$, and $\left(t_{4}, D_{4}\right)$, so that $t_{2}=t_{1}+\Delta t$, $t_{3}=t_{1}+2 \Delta t, t_{4}=t_{1}+3 \Delta t$.

Then

$$
\begin{aligned}
& e^{a\left(D_{1}-D_{2}\right)}=\frac{b-(b-\mathrm{I}) e^{-c t_{1}}}{b-(b-\mathrm{I}) e^{-c t_{1}} e^{-c \Delta t}}, \\
& e^{a\left(D_{1}-D_{3}\right)}=\frac{b-(b-\mathrm{I}) e^{-c t_{1}}}{b-(b-\mathrm{I}) e^{-c t_{1}} e^{-2 c \Delta t},} \\
& e^{a\left(D_{1}-D_{4}\right)}=\frac{b-(b-\mathrm{I}) e^{-c t_{1}}}{b-(b-\mathrm{I}) e^{-c t_{1}} e^{-3 c \Delta t}}, \\
& \frac{b-(b-\mathrm{I}) e^{-c t_{1}} e^{-c \Delta t}}{e^{a\left(D_{2}-D_{1}\right)}}=\frac{b-(b-\mathrm{I}) e^{-c t_{1}} e^{-2 c \Delta t}}{e^{a\left(D_{3}-D_{1}\right)}}=\frac{b-(b-\mathrm{I}) e^{-c t_{1}} e^{-3 c \Delta t}}{e^{a\left(D_{1}-D_{1}\right)}}, \\
& \frac{e^{a\left(D_{2}-D_{1}\right)}-e^{a\left(D_{4}-D_{1}\right)}}{e^{a\left(D_{2}-D_{1}\right)}-e^{a\left(D_{3}-D_{1}\right)}}=e^{-c \Delta t}, \\
& c=(\mathrm{I} / \Delta t) \log _{e} \frac{e^{a D_{2}}-e^{a D_{3}}}{e^{a D_{3}}-e^{a D_{4}}}, \\
& \left(e^{a D_{1}}-e^{a D_{2}}\right)\left(e^{a D_{3}}-e^{a D_{4}}\right)=\left(e^{a D_{2}}-e^{a D_{3}}\right)^{2}, \\
& b=\frac{e^{a D}-e^{-c t}}{\mathrm{I}-e^{-c t}} .
\end{aligned}
$$

These last three equations are sufficient to determine $a, b$, and $c$.

The practical difficulties connected with this method are quite material,

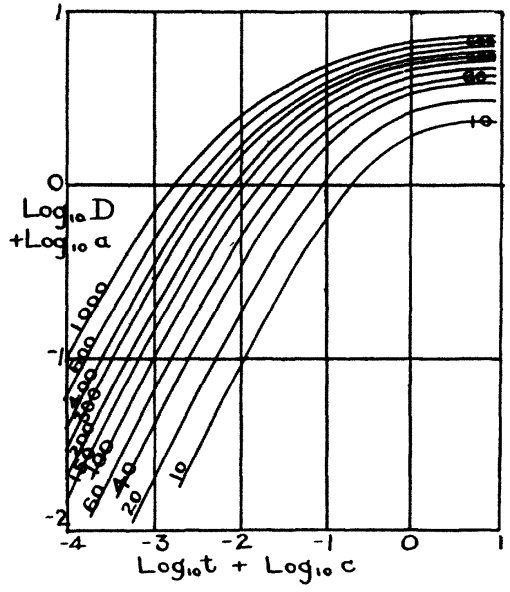

Fig. 1.

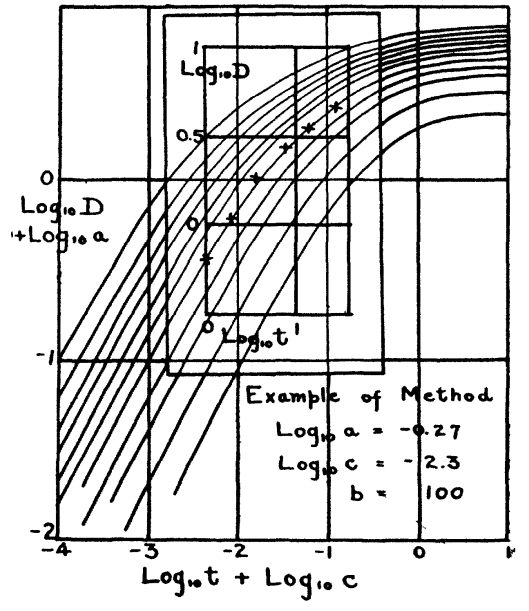

Fig. 2.

so a simple graphical method was accordingly developed.

A family of I I curves

$$
D=\mathrm{I} / a \log _{e}\left[b-(b-\mathrm{I}) e^{-c t}\right]
$$


was plotted as shown in the figure, with $b$ a parameter ranging in value from 10 to $I, 000$, as in Fig. I.

$\log a D$ was plotted along the $y$-axis, and $\log c t$ along the $x$-axis. The experimental curve was plotted upon tracing paper with $\log D$ and $\log t$ as ordinate and abscissa, respectively. The tracing paper was shifted over the family of curves preserving parallelism of axes, until it coincided with one of the family. $b$ was determined by the particular curve of the family which gave coincidence, and the $a$ and the $c$ were given by the intercepts of the axes of the experimental curve upon the axes of the family of curves. (Fig. 2.)

In actual practice, especially for large ranges of times of exposures, it was not difficult to find the position of the tracing paper which gave the optimum fit of the experimental curve to one of the theoretical curves,i.e., that the values of $a, b$, and $c$ are unique.

$\log c$ was next expressed as a function of Log $I$ by Campbell's "Method of the Zero Sum,"' 1 assuming that

$$
\log _{10} c=A+B \log _{10} I+C \log _{10}^{2} I \text {. }
$$

This relationship was selected because the curvature of the $\log c-$ $\log I$ curve was very small, and because the equation readily lends itself to methods of adjustment of constants, and to the determination of the exponent " $p$ " in Schwarzschild's "Law." $\left(I \cdot t^{p}\right.$ equals constant for equal blackening.)

In one case, namely the set of plates A-650, the curvature was more marked than in the other cases, and a term involving $\log _{10}{ }^{3} I$ was added to the expression.

The following values of the parameters were obtained by this method:

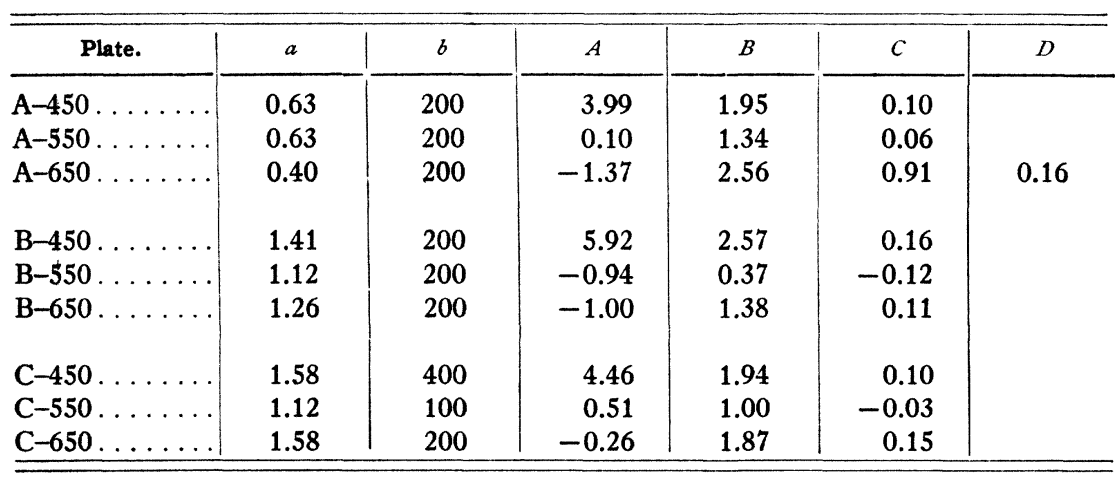

$$
\log _{10} c=A+B \log _{10} I+C \log _{10}{ }^{2} I+D \log _{10}{ }^{3} I .
$$

1 Phil. Mag., 39, 177; 1920.

${ }^{2}$ Loc. cit. 
In Fig. 3 the $\log _{10} c-\log _{10} I$ curves are plotted for the 9 different sets of plates. Points show values of " $c$ " determined graphically.

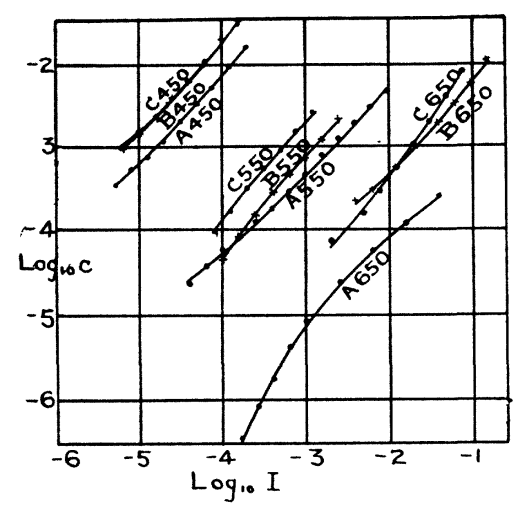

Fig. 3.

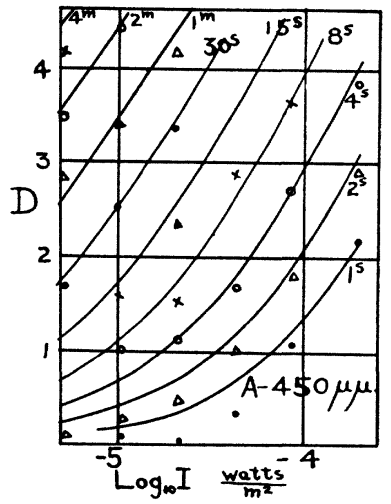

Fig. 4.

Curves show values of " $c$ " computed from

$$
\log _{10} C=A+B \log _{10} I+C \log _{10} 2 I .
$$

Figs. 4 to 12 show values of blackenings computed from the above

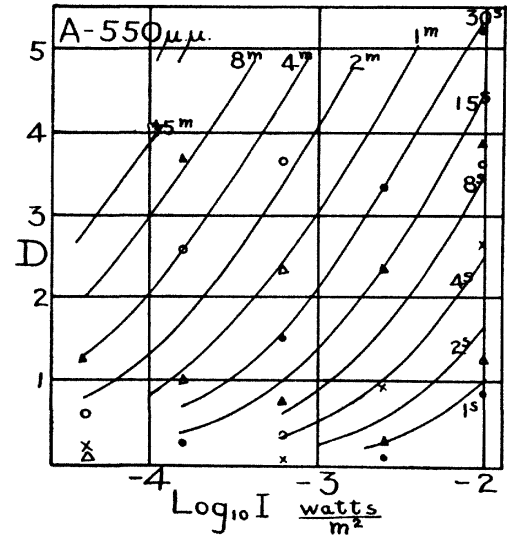

Fig. 5.

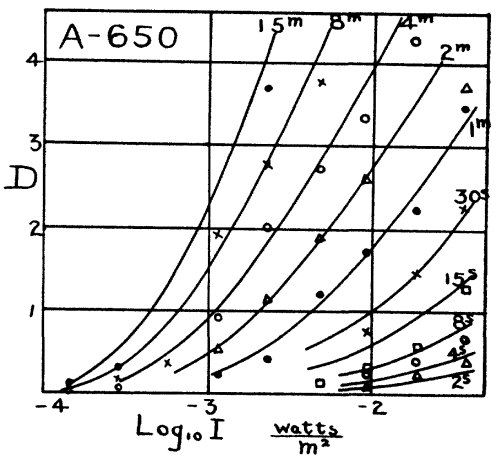

Fig. 6.

parameters, and the points represent the experimentally determined values.

The " $p$ " of Schwarzschild's so-called "Law" can be very easily obtained from the equation which the writer has used to express the relation between the density of a plate and the intensity of light and time of exposure.

Schwarzschild states that the product $I \cdot t^{p}$ must be constant for equal 
blackening, but Kron ${ }^{1}$ and Lemon ${ }^{2}$ have shown that $p$ is a function of the intensity of light.

Expressing the blackening of a plate in the form

where

$$
D=\mathbf{I} / a\left[\log _{e} b-(b-\mathbf{I}) e^{-c t}\right],
$$

$$
\log _{10} c=A+B \log _{10} I+C \log _{10} 2 I,
$$

then $c \cdot t$ must be constant for equal blackening, or

or

$$
t \cdot 10^{A+B \log _{10} I+C \log _{10} 2 I}=\text { constant. }
$$

$$
t \cdot I^{B+C \log _{10} I}=\text { constant. }
$$

So $\left(B+C \log _{10} I\right)^{-1}$ is equivalent to the " $p$ " of Schwarzschild's ex-

\begin{tabular}{|c|c|c|c|c|c|}
\hline \multirow{2}{*}{$\frac{\text { Plate. }}{A-450 \ldots}$} & \multicolumn{2}{|c|}{ Range of $\log _{10}$ Intensity. } & \multicolumn{3}{|c|}{ Range of $p$} \\
\hline & -3.7 & -5.3 & 1.09 & 1.01 & \\
\hline$A-550 \ldots$ & -2.0 & -4.4 & 1.22 & 1.06 & \\
\hline$A-650$. & -1.4 & $-3.8 \quad(-2.92$ Max. $)$ & 1.59 & 1.36 & (1.23 Max.) \\
\hline$B-450$. & -3.8 & -5.2 & 1.95 & 1.72 & \\
\hline B-550. . & -2.6 & -4.0 & 0.68 & 0.85 & \\
\hline B-650.. & -0.8 & -2.4 & 1.30 & 1.13 & \\
\hline $\mathrm{C}-450$. & -3.8 & -5.2 & 1.58 & 1.44 & \\
\hline C-550. . & -2.9 & -4.1 & 1.05 & 1.08 & \\
\hline C-650. & -1.1 & -2.7 & 1.71 & 1.48 & \\
\hline
\end{tabular}
pression.

The following extreme values of $p$ were computed in this manner, and show the variation in the value of $p$ with the intensity of light.

Procedure in Using Method for Photometric Work.

$$
\text { "Calibration" of Plates. }
$$

I. Plates are exposed to a known intensity of monochromatic light for various times of exposure and a $\log D-\log t$ curve plotted. Values of the constants $a$ and $b$ are determined from this curve with the aid of the monographic chart.

2. Plates are exposed for constant times of exposure to various known intensities of the monochromatic light and a $\log D-\log t$ curve plotted as in $\mathbf{I}$. Values of $c$ as a function of $I$ are now determined with the monographic chart.

3. $\log c$ is plotted against $\log I$.

${ }^{1}$ Loc. cit.

${ }^{2}$ Loc. cit. 


\section{Determination of Intensity.}

I. A plate is exposed for any length of time to the monochromatic light of unknown intensity.

2. Knowing the density of the plate, the time of exposure, and the

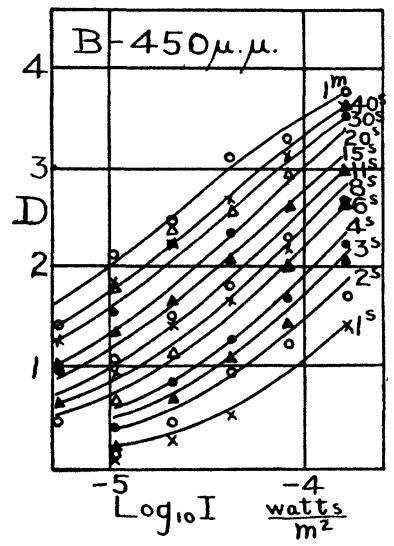

Fig. 7.

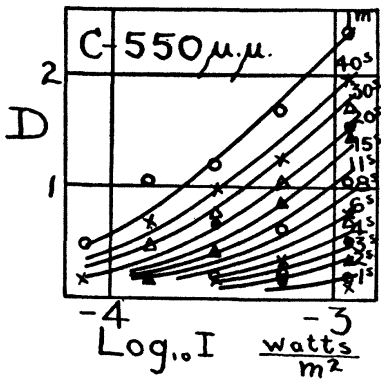

Fig. 8.

values of the constants $a$ and $b$, the value of $c$ for the particular intensity of light is determined from the monographic chart.

3. From the determined value of $c$, the value of $I$ is found from the $\log c-\log I$ curve.

\section{Some General Considerations.}

In plotting photographic blackening curves, the standard practice has always been to plot density of plate against logarithm of time of exposure, intensity being a parameter. In the light of the foregoing developments,

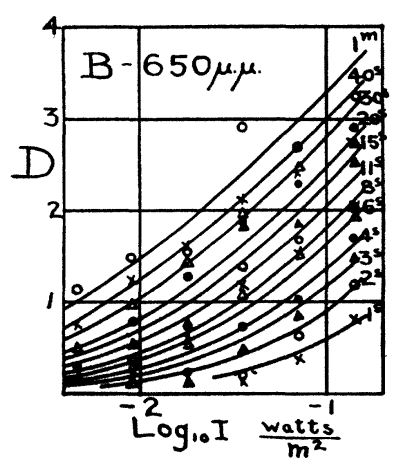

Fig. 9.

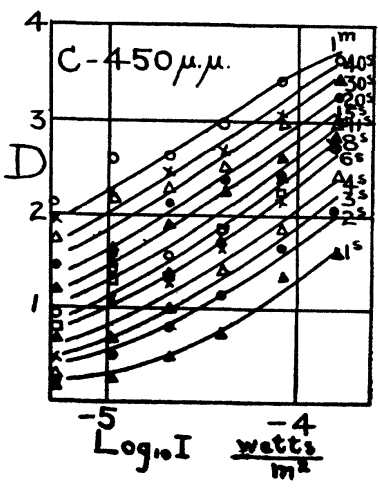

Fig. 10. 
as $a$ and $b$ are constant for a given set of curves, it would be more logical to plot logarithm of density against logarithm of time,- - for in that case the result would consist of a family of parallel curves. The actual drawing of such a family would be greatly facilitated, because the curves would be identical in shape. In this work however, the curves have

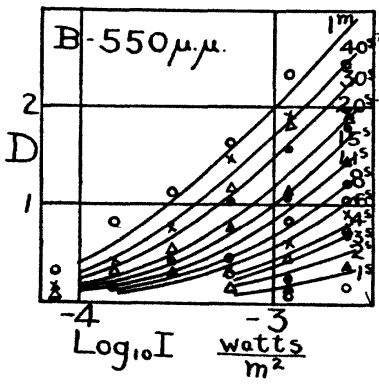

Fig. 11.

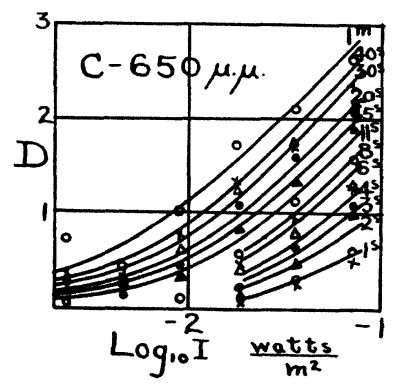

Fig. 12.

been plotted according to the standard practice in order that they may be comparable to previously published curves.

A set of exposures such as has been made throws much light upon the characteristics of fast and slow plates.

Consider first the relationship between brand of plate and blackening, with equal times of exposures and intensities of light, other factors being constant. In general, the curves of the slower plates are steeper than those of the faster ones.

The result is, that for a small intensity of light, the fast plate attains a greater density for a given time of exposure than does a slower plate. But for a longer time of exposure, the slower plate may attain a greater density than does the fast one. It is common knowledge among photographers that the longer the exposures, the less the difference that can be noticed between fast and slow plates.

The fact that the curves of the slow plates are steeper than those of the fast ones accounts for the greater contrast of a slow plate as compared to a fast one. With time of exposure constant, a small difference of intensity of light on the slow plate means a much greater difference in density in the slow plate than in the fast one.

The effect of wave-length of light upon the blackening, with intensity and time constant, is of course, very apparent, but the relation between slope of curves with wave-length of light is not marked. In fact, Leimbach $^{1}$ claims, though others have questioned his conclusions, that the slope, or "gradation" is independent of the wave-length. Upon closer

${ }^{1}$ Zeit. wiss. Phot., 7, I57 u. I81; 1909. 
inspection, the writer's curves show a tendency to have the least gradation for that wave-length where the plate has its greatest sensibility, as Abney, ${ }^{1}$ Stark $^{2}$ and others have found.

Certain generalizations can also be made with regard to the $\log c$ - Log $I$ curves. First, the curves depart more or less from a straight line. If Schwarzschild's expression were valid,-i.e., $I \cdot t^{p}=$ constant for equal blackening, the $\log c-\log I$ curves would be straight lines, as may be proved from the expression $p=\left(B-C \log _{10} I\right)^{-1}$. The curves may therefore be regarded as giving some indication of the amount of error which is involved in assuming Schwarzschild's expression.

If Bunsen and Roscoe's "Law" held,-i.e., $I \cdot t=$ constant for equal blackening, the curves would be straight lines with slope equal to unity, or $c$ would equal $I$ (Hurter and Driffield's assumption). The curves show that this is only a very rough approximation.

\section{Conclusions.}

Two methods of obtaining the mathematical equation of the blackening of a photographic plate as a function of intensity of monochromatic light and time of exposure have been pointed out.

Using the method of the monographic chart, the blackening equations of three different brands of plates have been calculated for three different wave-lengths of light. In these nine different cases, there is fairly good agreement between the experimental and the calculated values.

It is a pleasure to acknowledge the encouragement received from the department of physics of the State University of Iowa, and particularly from Professors L. P. Sieg, and H. L. Dodge, the latter now of the University of Oklahoma.

Ross $^{3}$ has pointed out an apparent inconsistency in the assumptions underlying the Hurter and Driffield equation made use of in the formulae above, and as a substitute develops the equation

$$
D=d_{m}\left[\mathrm{I}=\frac{\mathrm{I}}{n} \sum_{s=0}^{s=n-1} e^{-r * k I t}\right]
$$

$D$ is the density of the plate, $d_{m}$ the density for infinite exposure, $I$ the intensity of light (assumed monochromatic, $t$ the time of exposure, and $k, r$, and $n$ are certain parameters. A method is given of determining $n$ if $d_{m}$ can be found by experiment, if arbitrary values assigned to $r$ and $k$. The expression will hold only for one intensity of light unless the Reciprocity "Law" ( $I \cdot t=$ constant for equal blackening) be assumed.

\footnotetext{
1 Treatise on Photography, roth Ed. Longmans, p. 413.

2 Ann. der Phys., 35, 474; I9I1.

${ }^{3}$ Journ. Optical Soc, of Am., 4, 261 ; September, I920.
} 
Ross' formula can be made the basis of expressing the blackening of a plate exposed to monochromatic light as a function of intensity of light and time of exposure according to the graphical method already outlined above if the formula is written in the form

$$
D=\frac{\mathrm{I}}{a}\left[\mathrm{I}-\frac{\mathrm{I}}{n} \sum_{s=0}^{s=n-1} e^{-r s c t}\right],
$$

and families of $\log a D-\log c t$ curves are plotted with "and $r$ as parameters.

A set of experimental $\log D-\log t$ curves is obtained ( $I$ having a different value for each curve), and a particular $\log a D-\log c t$ curve is chosen so that the $n, r$, and $a$ will give a good fit to each of the Log $D-\log t$ curves by allowing $c$ to vary. The $c$ and the $a$ are found from the $x$ and the $y$-intercepts of the axes of the $\log D-\log t$ curves on the axes of the $\log a D-\log c t$ axes, and the $n$ and the $r$ are determined by the particular $\log a D$ - Log $c t$ curve chosed to give the best fit. If $c$ be next expressed as a function of $I$, we have a mathematical expression for the blackening of a photographic plate as a function of the intensity of monochromatic light and time of exposure.

The possible advantages of a graphical means of determining the parameters of Ross' equation seems to lie in the fact that by a graphical method the reader can easily judge the accuracy of fit of the experimental to the theoretical curve, and can make the curves fit particularly well at any desired point, that he can find at once the values of the parameters $r, n$, and $k$, and that he is not limited to using one particular value of $r$ or $k$.

Physical Laboratory,

The State University of Iowa,

June, 1920. 